\title{
Avaliações preliminares da permeabilidade de um arenito a partir do AutoLab 1000
}

\author{
Arthur Chornobay, Darby Pereira Dantas de Lima, Luiz Fernando Cursino Tabosa e Adriana Chatack Carmelo
}

Copyright 2019, SBGf - Sociedade Brasileira de Geofísica

This paper was prepared for presentation during the $16^{\text {th }}$ International Congress of the Brazilian Geophysical Society held in Rio de Janeiro, Brazil, 19-22 August 2019.

Contents of this paper were reviewed by the Technical Committee of the $16^{\text {th }}$ International Congress of the Brazilian Geophysical Society and do not necessarily represent any position of the SBGf, its officers or members. Electronic reproduction or storage of any part of this paper for commercial purposes without the written consent of the Brazilian Geophysical Society is prohibited.

\section{Abstract}

This study presents the results of two different permeability measurements with AutoLab 1000 for the same sample of sandstone. The use of complex transients as ASPIKE, Multipulse and SINE6 complex allows evaluating and comparing the permeability results in relation to confining pressure and pore pressure applied. The sandstone sample belongs to the Urucuia Group, Jalapão-TO. It is observed that part of this sample is cemented. This led to two-step experiments, High Permeability and Low Permeability acquisition for comparisons.

\section{Introdução}

Esta pesquisa apresenta o uso do AutoLab 1000 na análise de permeabilidade para identificar a caracterizar pressão de poro e as mudanças efetivas em aplicação de pressões distintas. A amostra analisada é um arenito (Grupo Urucuia, TO), rocha armazenadora em potencial. A estimativa da pressão de poro e o conhecimento das propriedades do espaço poroso da rocha (porosidade e permeabilidade) são fatores importantes na indústria de petróleo e gás para avaliação de reservatórios e de aquíferos.

O objetivo principal deste trabalho é a comparação da resposta de permeabilidade a partir da aplicação de transientes complexos ASPIKE, Multipulse e SINE6 em pressões distintas. Neste trabalho, foram utilizadas pressões confinantes máximas de $60 \mathrm{MPa}$ e pressões de poro máximas de 7MPa para amostras classificadas com potencial para alta permeabilidade. Para amostras com baixa permeabilidade, as pressões confinantes máximas são $10 \mathrm{MPa}$ e as pressões de poro máximas de $5 \mathrm{Mpa}$.

\section{Materiais}

Para a realização dos experimentos de permeabilidade das rochas foi utilizado o equipamento AutoLab 1000, da New England Research. O equipamento é um sistema servo-hidráulico hidrostático utilizado para a medição da propriedade do espaço poroso, permeabilidade, e de parâmetros geofísicos: compressão e velocidades das ondas de cisalhamento, resistividade, compressibilidade do volume de poros e compressibilidade linear de amostras de rochas de até $50 \mathrm{~mm}$ de diâmetro na pressão de sobrecarga in situ, pressão de poros e temperatura.

O método de medição envolve o controle de transientes complexos na pressão de poro na parte superior amostra (upstream) enquanto monitora a resposta na extremidade inferior (downstream). A permeabilidade é calculada ao ajustar a resposta com soluções analíticas, para amostras de alta e baixa permeabilidades em milidacies $(\mathrm{mD})$, nanodarcies $(\mathrm{nD})$ e microdarcies $(\mu \mathrm{D})$.

A amostra de rocha utilizada foi um arenito do Jalapão (TO) pertencente ao grupo Urucuia, com diâmetro e altura de $25 \mathrm{~mm}(1 \mathrm{ln})$ (Figura 1). É composta por quartzo $(82 \%)$ e feldspato (2\%) muito alterado, com pseudomatriz formada por produtos da alteração de feldspato com opacos (16\%). Os contatos entre os grãos são predominantemente dos tipos planar e, em alguns casos, côncavo-convexo, indicando ação de compactação mecânica. Os poros são formados por dissolução da matriz e têm dimensões com diâmetro de $>0,1 \mathrm{~mm}$ a $1 \mathrm{~mm}$, com formas variadas. A rocha apresenta porosidade de aproximadamente $12 \%$, mas por serem poros de dissolução (pontuais), estima-se que a permeabilidade é baixa (Bergman 2017)

Os softwares utilizados foram: i) AutoLAB6, utilizado para a aquisição dos dados, criação do projeto, modificação das pressões confinante $(\mathrm{Pc})$ e de poros $(\mathrm{Pp})$, escolha dos transientes e geração dos gráficos; e ii) AutoLAB viewer, utilizado para visualizar e exportar os gráficos obtidos durante a aquisição dos dados. 


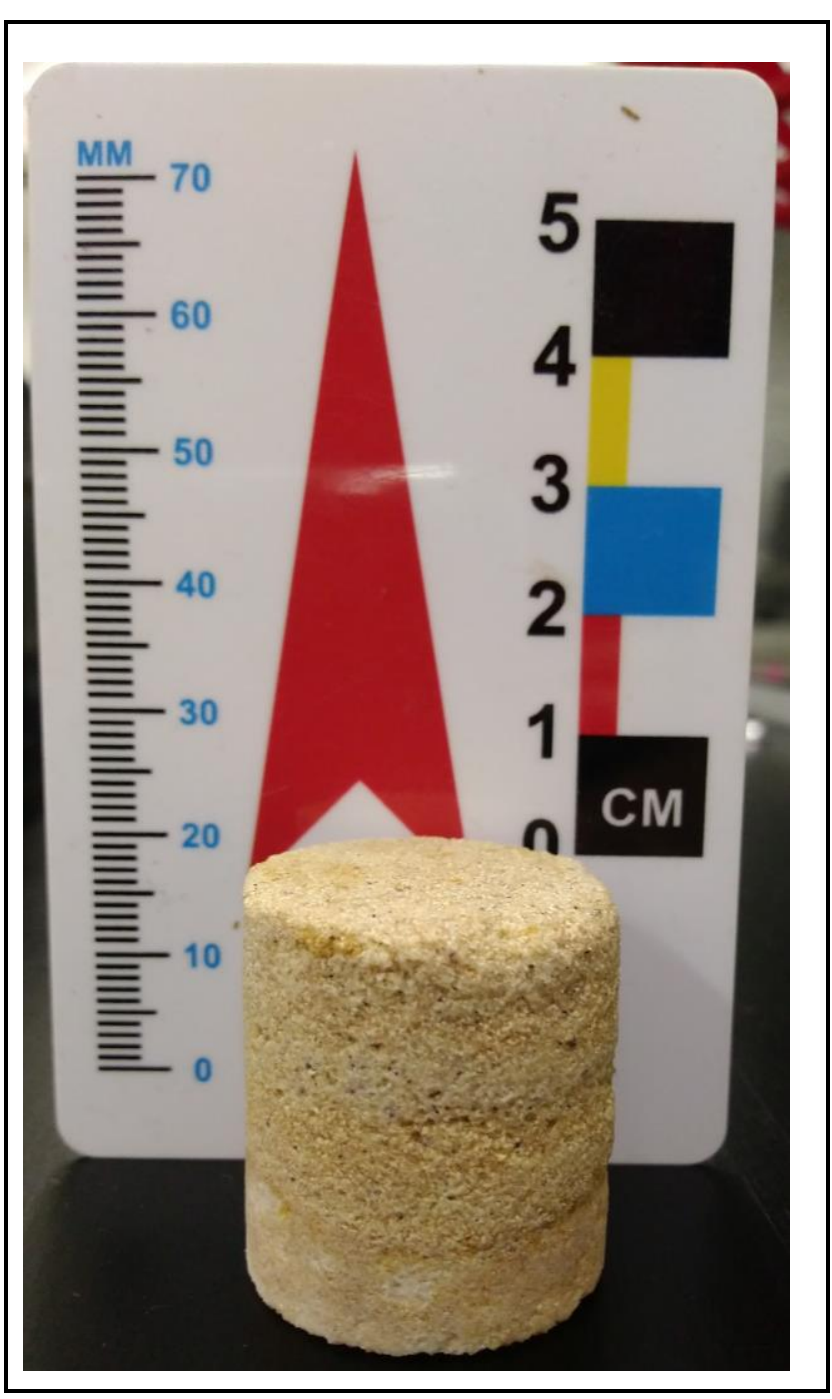

Figura 1 - Amostra do arenito do Grupo Urucuia, Jalapão-TO.

\section{Métodos}

A metodologia empregada nas medições de permeabilidade utiliza os transientes complexos tanto para rochas de alta permeabilidade como de baixa permeabilidade. A resposta do sistema com o uso de transientes complexos é simplificada e torna-se insensível à capacidade de armazenamento da amostra o que torna as medidas mais confiáveis e robustas. A resposta da pressão $(P)$ para uma função degrau em pressão de amplitude $\left(P_{0}\right)$ em um tempo $t=0$ segue:

$$
P=P_{0}\left(1-e^{-\frac{k t}{m}}\right) \text { (Eq. 1) }
$$

Onde $(\mathrm{m})$ é uma constante dependente do fluido e das propriedades do sistema e da geometria da amostra; (k) é a permeabilidade. De maneira similar à perturbação senoidal, a função de transferência para esse caso em especial pode ser reescrita no domínio da frequência como:

$$
A_{T}=\left(1+\omega^{2} m^{2} k^{2}\right) \text { (Eq. 2) }
$$

$$
\phi=-\arctan \left(\frac{k}{\omega m}\right) \text { (Eq. 3) }
$$

Onde ( $A_{\Upsilon}$ ) é a razão da amplitude, $(\Phi)$ é a mudança de fase e a frequência angular $(\omega)$. Para a senoidal, a permeabilidade pode ser medida utilizando ainda a razão da amplitude ou a mudança de fase.

Nesta pesquisa foram utilizados transientes complexos ASPIKE, Multipulse e SINE6.

O transiente ASPIKE possui banda larga e carrega energia de alta frequência significativa. Essa característica torna-o ideal para medidas realizadas em rochas que apresentam de alta permeabilidade, mas também é aplicada em amostras de rochas com baixa permeabilidade.

De maneira semelhante ao transiente ASPIKE, o transiente Multipulse é medido quando é o aplicado em diferentes taxas de fluxo com tempo de duração de cada pulso dependente do tempo. É útil para realização de testes rápidos e pequeno tempo de cálculo.

O transiente SINE6 é a soma de 6 senoidais de amplitude idêntica em 6 diferentes frequências estendendo-se como função senoidal simples. Sua limitação é decorrente de frequências mais elevadas. Como vantagem, comporta-se como função 'quasecontínua' e em princípio pode apresentar baixo erro, dependendo da amostra analisada (Boitnott, 1997).

O AutoLab 1000 possui diferentes modos para a aquisição conforme a permeabilidade das rochas, caso a rocha seja de alta permeabilidade utiliza-se um volume de pressão de poro, comparativo, alto. Caso a amostra seja de média permeabilidade, esse volume de pressão de poro diminui para $2 \%$ do volume utilizado em rochas de alta permeabilidade. Assim, se a rocha possui baixa permeabilidade o volume de pressão de poro é reduzido para $0,03 \%$ do volume utilizado em rochas de alta permeabilidade.

\section{Resultados}

A amostra do arenito do Grupo Urucuia apresentou um resultado fora do esperado inicialmente, dessa forma, foram aplicados dois métodos de medições para a mesma amostra, conforme o AutoLab 1000 apresenta: para alta permeabilidade (High Perm) e baixa permeabilidade (Low Perm).

Inicialmente, esperava-se uma resposta de alta permeabilidade, pois a rocha é um arenito e potencialmente apresenta boa permeabilidade. Entretanto, os resultados mostraram baixa compatibilidade matemática conforme apresentado nas figuras 2 e 3 . Para a aquisição desses resultados foi utilizada a configuração de pressão confinante $(P c)$ em $30 \mathrm{MPa}$ e pressão de poro (Pp) $7 \mathrm{MPa}$, ademais, a amplitude foi de $1 \mathrm{MPa}$ e a frequência de $0,5 \mathrm{~Hz}$. Por fim, utilizou-se duas funções matemáticas para a aplicação do transiente, ASPIKE e Multipulse, respectivamente, com valores de permeabilidade $\mathrm{k}=0.000548 \mathrm{mD}$ (storage $=0 \%$ ) e $\mathrm{k}=0.0567 \mathrm{mD}$ (storage $=0 \%$ ). 


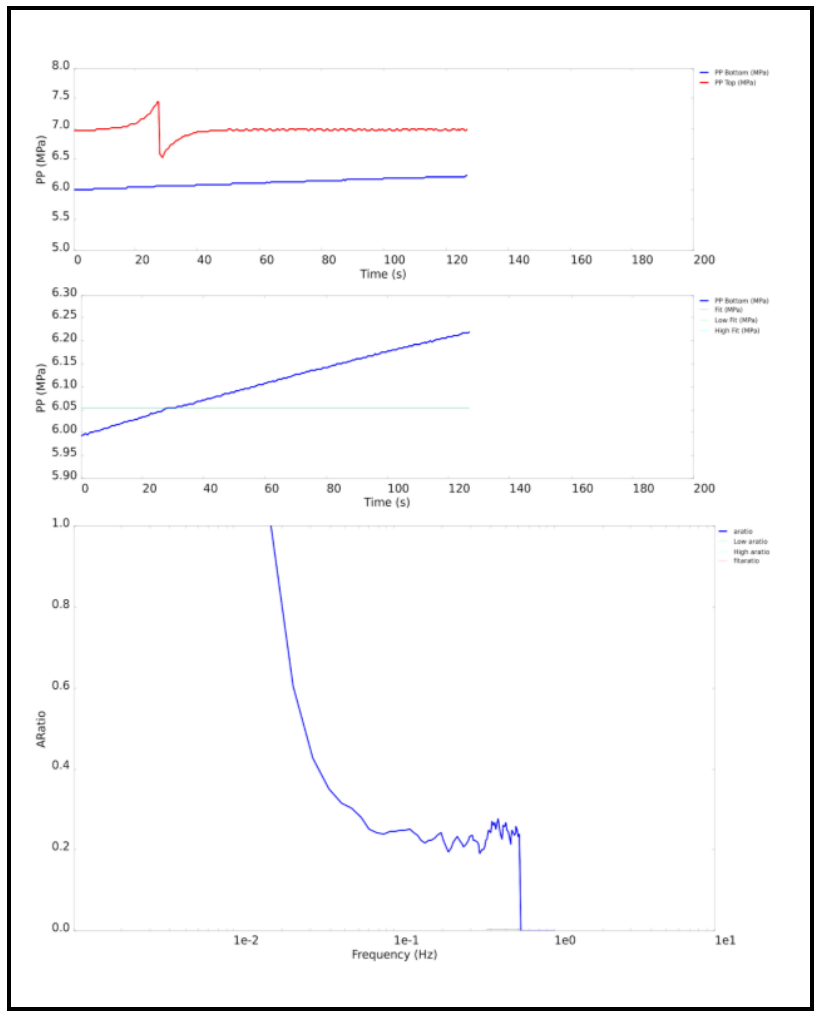

Figura 2 - Gráficos de resultados ASPIKE do arenito, Grupo Urucuia (Jalapão-TO), utilizando o método de alta permeabilidade.

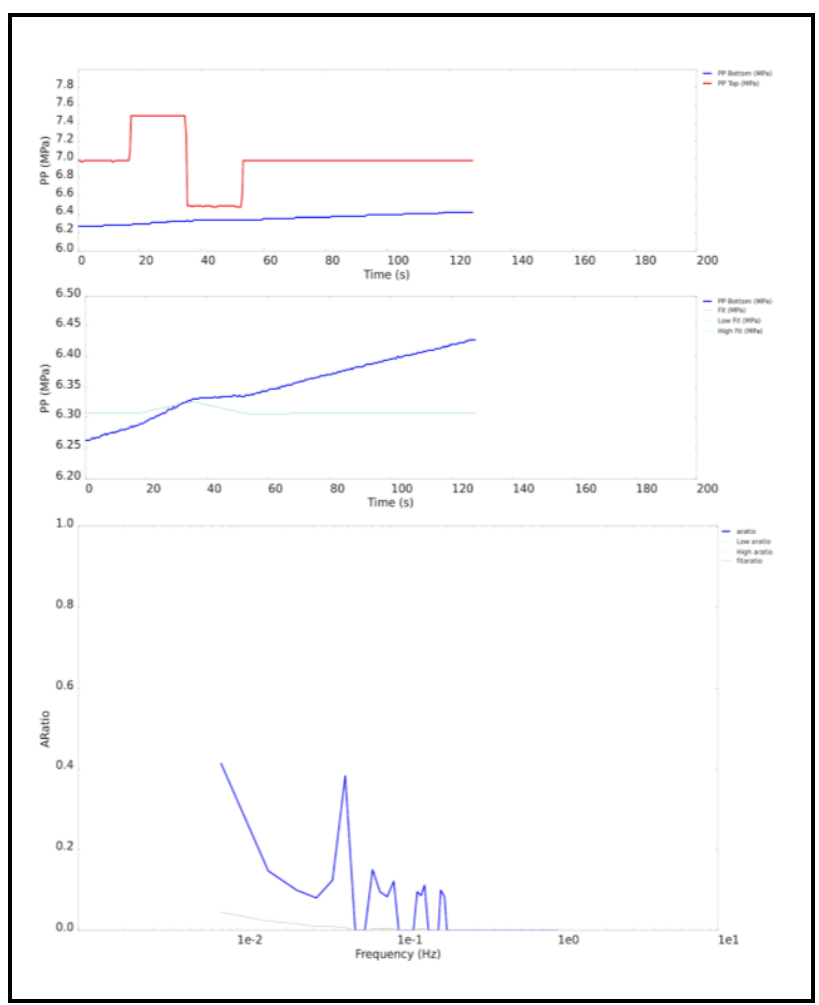

Figura 3 - Gráficos de resultados Multipulse do arenito, Grupo Urucuia (Jalapão-TO), utilizando o método de alta permeabilidade.
Para a segunda aquisição de dados, utilizou-se o método de aquisição para rochas de baixa permeabilidade (Low Perm), e a configuração utilizada foi de pressão confinante $(\mathrm{Pc})$ em $7 \mathrm{MPa}$ e pressão de poro $(\mathrm{Pp})$ em $4 \mathrm{MPa}$, com amplitude de $1 \mathrm{MPa}$ e frequência de $0,5 \mathrm{~Hz}$. Por fim, utilizou-se três funções matemáticas para a aplicação do transiente: a ASPIKE, a Multipulse e a SINE6.

Os valores de permeabilidade ( $k$ ) obtidos foram: i) ASPIKE: $k=0,00195 \mathrm{mD}$ (storage $=0,785 \%$ ); ii) Multipulse: $\mathrm{k}=0,00176 \mathrm{mD} \quad$ (storage $=0,344 \%) ; \quad$ e iii) SINE6: $\mathrm{k}=0,00171 \mathrm{mD}$ (storage $=0,308 \%$ ). Dessa forma, os resultados obtidos são matematicamente bem ajustados, conforme apresentados nas figuras 4 a 9.

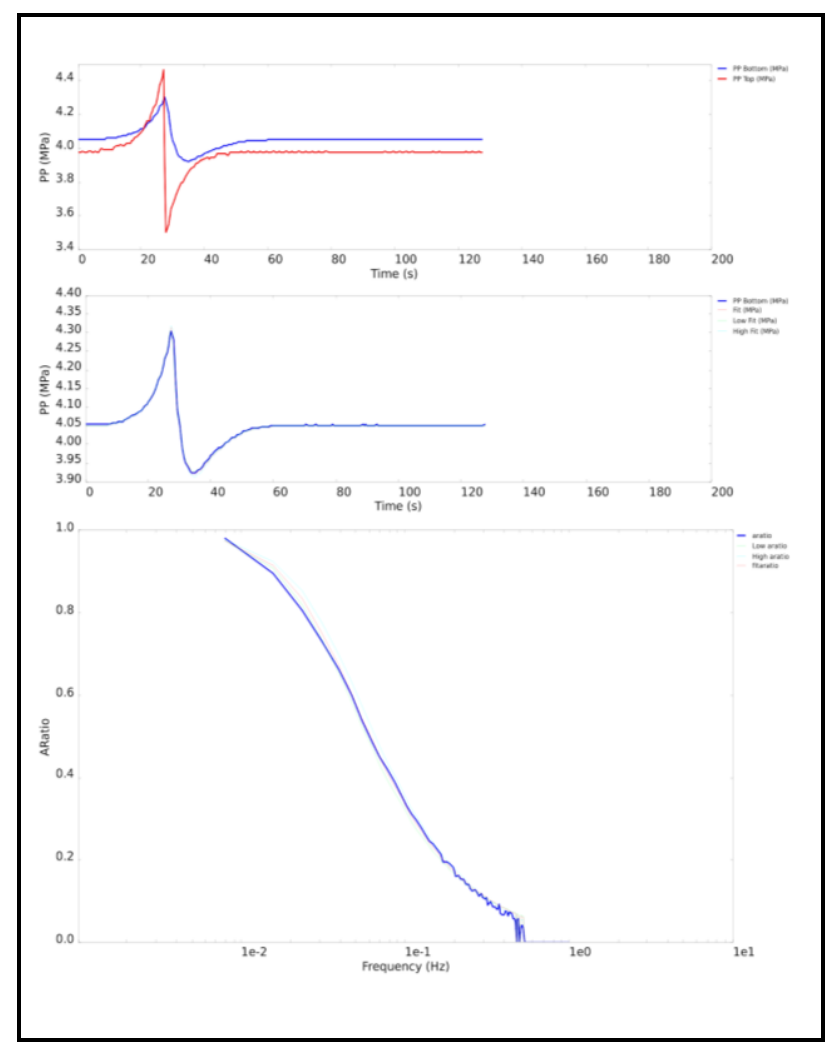

Figura 4 - Gráficos de resultados ASPIKE do arenito, Grupo Urucuia (Jalapão-TO), utilizando o método de baixa permeabilidade. 


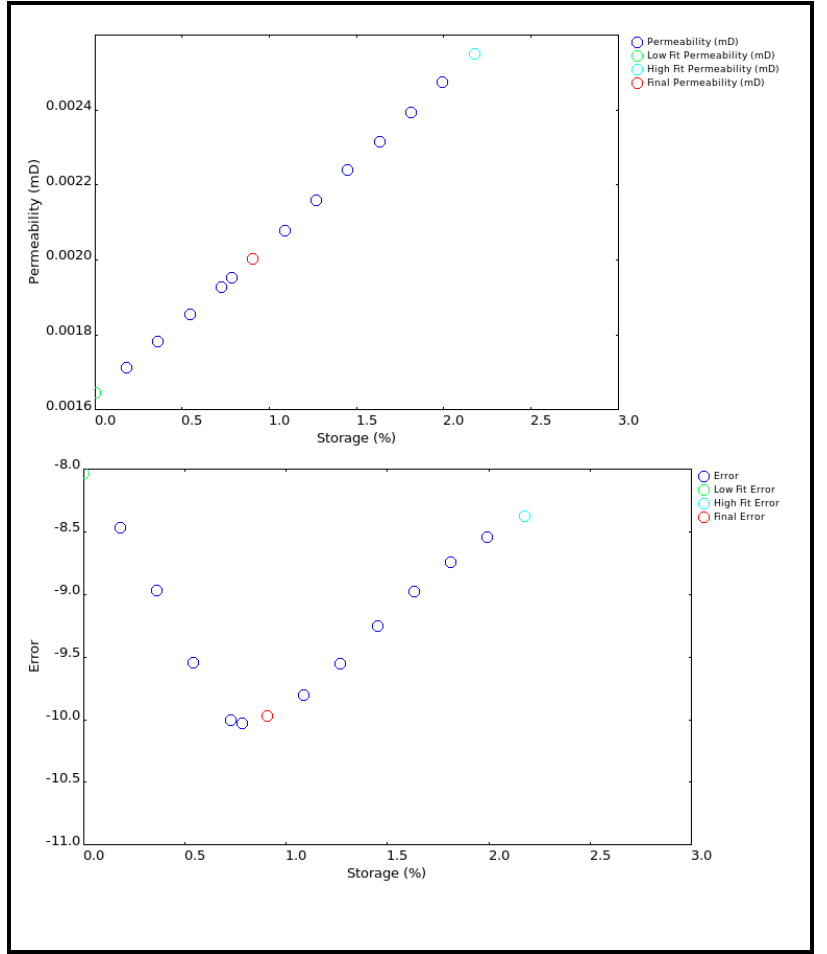

Figura 5 - Gráficos da permeabilidade $(m D)$ e do erro associado à permeabilidade, ambos, em função do armazenamento (\%), gerados pelo transiente ASPIKE.

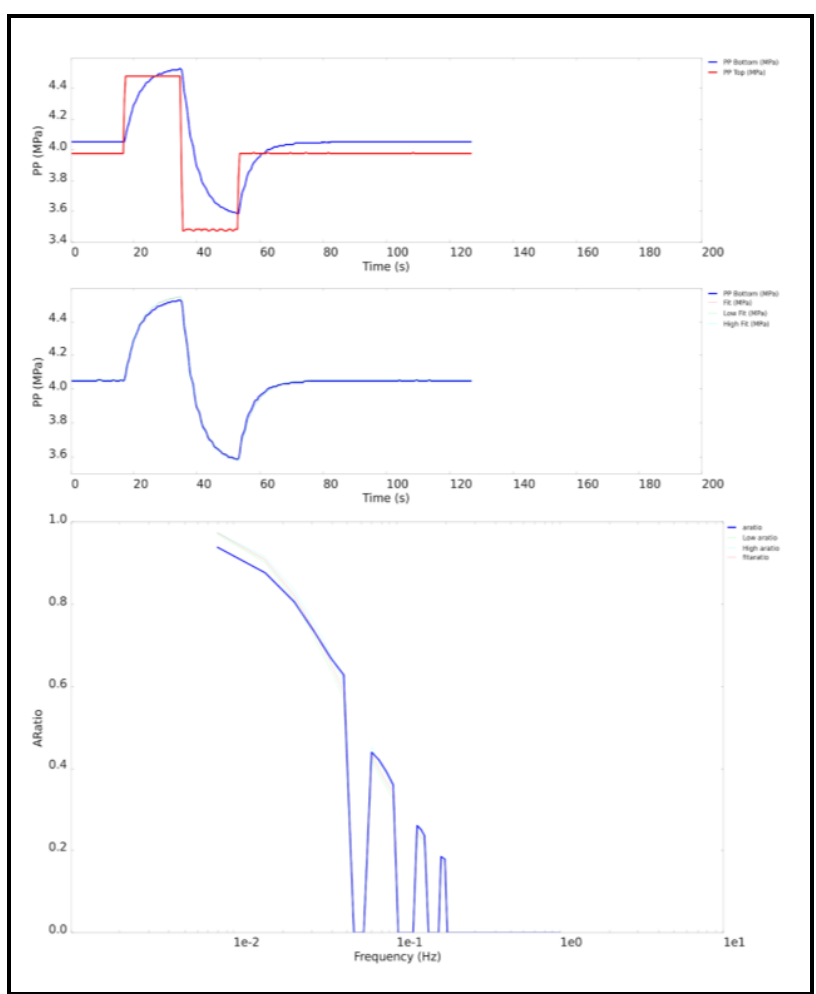

Figura 6 - Gráficos de resultados Multipulse do arenito, Grupo Urucuia (Jalapão-TO), utilizando o método de baixa permeabilidade.

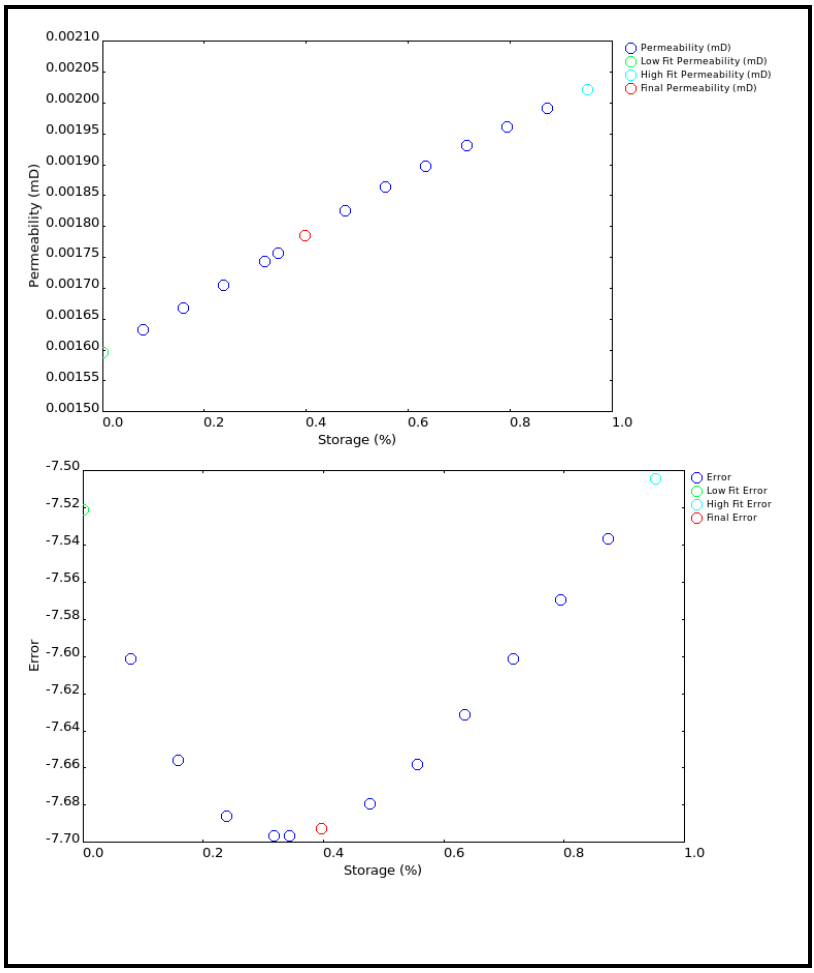

Figura 7 - Gráficos da permeabilidade $(m D)$ e do erro associado à permeabilidade, ambos, em função do armazenamento (\%), gerados pelo transiente Multipulse.

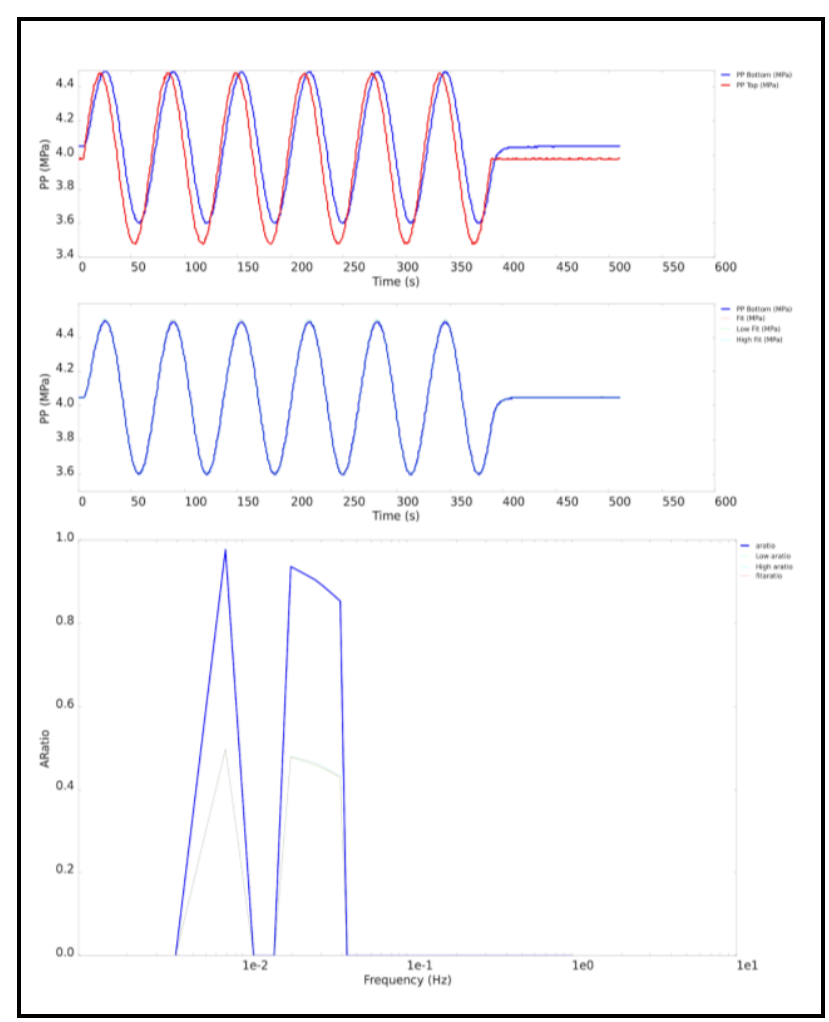

Figura 8 - Gráficos de resultados Sine6 do arenito, Grupo Urucuia (Jalapão-TO), utilizando o método de baixa permeabilidade. 


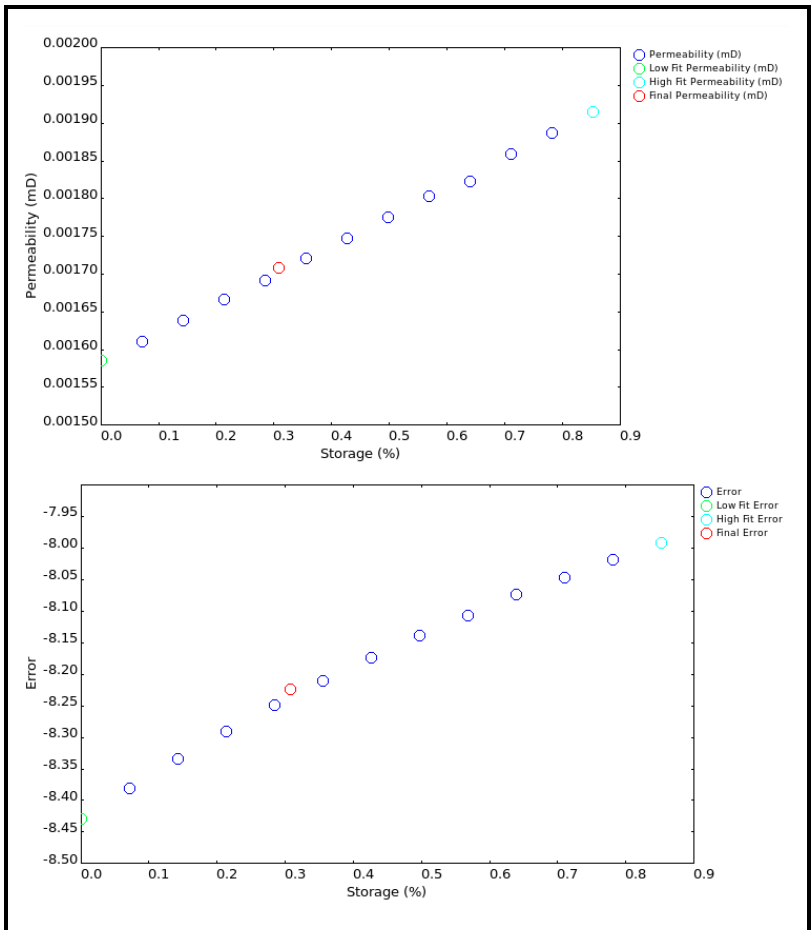

Figura 9 - Gráficos da permeabilidade $(m D)$ e do erro associado à permeabilidade, ambos, em função do armazenamento (\%), gerados pelo transiente SINE6.

\section{Conclusões}

Os dados obtidos por meio dos experimentos mostram a eficácia dos métodos de aquisição do AutoLab 1000, uma vez que a resposta de permeabilidade da amostra do arenito do Jalapão foi bem ajustada em resultados de baixa permeabilidade, pois a amostra é um arenito que possui heterogeneidade em sua composição e apresenta cimentação em cerca de um terço da amostra, influenciando diretamente na propriedade física do espaço poroso denominada permeabilidade.

\section{Agradecimentos}

- Laboratório de Propriedades Físicas das Rochas, Instituto de Geociências da Universidade de Brasília.

- Steve Henck e André Felipe.

- NER - New England Research Inc..

- Tennessine - Instrumentação Analítica.

\section{Referências}

Bergman, S. S., 2017. Microtomografia computadorizada por raios $\mathrm{X}$ aplicada às rochas: avaliação da porosidade $\mathrm{e}$ aspectos texturais. Monografia de conclusão do curso de Geofísica. Instituto de Geociências da Universidade de Brasília, Brasil, 107p
Boitnott, G., 1997. Use of Complex Pore Pressure Transients to Measure Permeability of Rocks. In: SPE International- Society of Petroleum Engineers., 38-45. 